Supporting information for

\title{
Atmospheric Concentrations and Deposition of PCBs to the Hudson River Estuary
}

Lisa A. Totten, Cari L. Gigliotti, Daryl A. VanRy, John H. Offenberg, Eric D. Nelson, Jordi Dachs, John R. Reinfelder, and Steven J. Eisenreich

Five pages (including cover page ) containing four tables detailing annual average concentrations and deposition fluxes at all nine sites listed by congener and homolog group

Abbreviations:

$\begin{array}{ll}\text { CC } & \text { Camden } \\ \text { DB } & \text { Delaware Bay } \\ \text { JC } & \text { Jersey City } \\ \text { NB } & \text { New Brunswick } \\ \text { PL } & \text { Pinelands } \\ \text { SH } & \text { Sandy Hook } \\ \text { TK } & \text { Tuckerton } \\ \text { WC } & \text { Washington Crossing } \\ \text { CH } & \text { Chester }\end{array}$


Table S1: Annual average gas-phase PCB concentrations at NJADN sites

Annual average concentrations $\left(\mathrm{pg} / \mathrm{m}^{3}\right)$

\begin{tabular}{|c|c|c|c|c|c|c|c|c|c|}
\hline site & CC & DB & JC & NB & PL & SH & TK & WC & $\mathbf{C H}$ \\
\hline PCB congener & gas & gas & gas & gas & gas & gas & gas & gas & gas \\
\hline 18 & 157 & 14 & 88 & 37 & 12 & 30 & 12 & 18 & 11 \\
\hline $17+15$ & 90 & 0.79 & 84 & 25 & 11 & 17 & 4.4 & 8.5 & 4.7 \\
\hline $16+32$ & 150 & 8.1 & 92 & 43 & 11 & 30 & 16 & 17 & 10 \\
\hline 31 & 222 & 11 & 88 & 36 & 11 & 29 & 13 & 16 & 10 \\
\hline 28 & 140 & 6.2 & 62 & 27 & 6.5 & 20 & 8.4 & 8.7 & 6.4 \\
\hline $21+33+53$ & 75 & 0.50 & 41 & 20 & 3.3 & 12 & 5.8 & 3.1 & 1.4 \\
\hline 22 & 91 & 4.0 & 34 & 22 & 5.3 & 20 & 6.5 & 7.4 & 5.3 \\
\hline 45 & 15 & 0.81 & 15 & 7.4 & 1.2 & 6.9 & 0.92 & 1.5 & 1.0 \\
\hline $52+43$ & 263 & 8.2 & 68 & 31 & 8.6 & 31 & 16 & 12 & 10 \\
\hline 49 & 81 & 5.7 & 35 & 17 & 6.5 & 16 & 9.0 & 9.2 & 6.8 \\
\hline $47+48$ & 47 & 3.3 & 23 & 16 & 3.0 & 15 & 3.6 & 3.9 & 2.7 \\
\hline 44 & 149 & 4.8 & 44 & 21 & 5.5 & 19 & 6.8 & 10 & 6.2 \\
\hline $37+42$ & 52 & 2.7 & 25 & 7.9 & 2.9 & 10 & 3.4 & 4.3 & 3.5 \\
\hline $41+71$ & 60 & 2.1 & 24 & 8.8 & 2.4 & 9.9 & 3.0 & 3.3 & 2.9 \\
\hline 64 & 39 & 1.3 & 14 & 6.4 & 1.5 & 5.7 & 1.9 & 2.2 & 1.8 \\
\hline 74 & 48 & 1.6 & 13 & 8.5 & 1.3 & 6.4 & 2.5 & 1.9 & 1.6 \\
\hline $70+76$ & 115 & 2.6 & 24 & 17 & 2.7 & 11 & 3.3 & 3.5 & 3.2 \\
\hline $66+95$ & 338 & 9.8 & 74 & 38 & 8.4 & 38 & 13 & 11 & 10 \\
\hline 91 & 33 & 0.73 & 9.0 & 8.3 & 0.69 & 6.6 & 0.61 & 1.8 & 0.84 \\
\hline $56+60+89$ & 75 & 2.3 & 18 & 11 & 2.0 & 7.7 & 2.6 & 2.7 & 2.5 \\
\hline $92+84$ & 142 & 5.8 & 29 & 17 & 4.6 & 12 & 6.4 & 5.5 & 4.9 \\
\hline 101 & 173 & 5.2 & 28 & 16 & 4.1 & 13 & 5.5 & 4.8 & 5.2 \\
\hline 97 & 35 & 1.2 & 5.6 & 4.0 & 0.90 & 3.0 & 1.2 & 0.99 & 1.1 \\
\hline $87+81$ & 50 & 0.78 & 10 & 7.5 & 1.6 & 5.3 & 2.9 & 0.98 & 0.79 \\
\hline $85+136$ & 30 & 0.98 & 6.7 & 6.8 & 0.60 & 2.9 & 1.1 & 0.78 & 0.64 \\
\hline $110+77$ & 167 & 5.2 & 25 & 15 & 3.2 & 13 & 4.5 & 4.2 & 4.7 \\
\hline 82 & 16 & 0.36 & 2.0 & 1.1 & 0.24 & 0.92 & 0.31 & 0.30 & 0.36 \\
\hline 151 & 16 & 1.7 & 4.7 & 2.2 & 0.83 & 1.8 & 1.4 & 0.95 & 0.99 \\
\hline $135+144+147+124$ & 24 & 1.5 & 5.0 & 2.6 & 0.71 & 2.0 & 1.0 & 0.74 & 0.99 \\
\hline $149+123+107$ & 65 & 4.0 & 14 & 6.5 & 1.7 & 5.3 & 2.8 & 2.0 & 2.3 \\
\hline 118 & 40 & 0.79 & 7.0 & 4.5 & 0.60 & 3.4 & 2.0 & 0.51 & 0.36 \\
\hline $153+132$ & 62 & 5.4 & 13 & 5.7 & 1.8 & 5.5 & 3.0 & 1.9 & 2.2 \\
\hline $163+138$ & 76 & 7.1 & 13 & 6.5 & 1.7 & 5.5 & 2.8 & 1.9 & 2.3 \\
\hline $187+182$ & 12 & 2.2 & 3.4 & 1.9 & 0.54 & 1.9 & 1.2 & 0.46 & 0.49 \\
\hline 180 & 7.2 & 3.7 & 3.0 & 1.2 & 0.61 & 1.1 & 0.81 & 0.42 & 0.45 \\
\hline 201 & 15 & 1.3 & 1.3 & 0.68 & 0.29 & 0.55 & 0.75 & 0.26 & 0.23 \\
\hline 203+196 & 14 & 1.5 & 1.6 & 0.62 & 0.36 & 0.55 & 0.77 & 0.30 & 0.27 \\
\hline 206 & 2.2 & 0.14 & 0.16 & 0.069 & 0.045 & 0.054 & 0.086 & 0.033 & 0.033 \\
\hline \multicolumn{10}{|l|}{ Homologue Group } \\
\hline 3 & 976 & 47 & 469 & 214 & 61 & 168 & 68 & 83 & 53 \\
\hline 4 & 907 & 34 & 314 & 174 & 36 & 160 & 50 & 52 & 40 \\
\hline 5 & 1085 & 33 & 143 & 77 & 27 & 74 & 40 & 32 & 31 \\
\hline 6 & 316 & 26 & 58 & 25 & 9.5 & 24 & 16 & 11 & 12 \\
\hline 7 & 40 & 13 & 14 & 6.5 & 2.4 & 5.8 & 4.6 & 2.2 & 2.2 \\
\hline 8 & 46 & 6.4 & 6 & 1.9 & 1.2 & 1.8 & 2.5 & 1.1 & 0.97 \\
\hline 9 & 2.2 & 0.14 & 0.16 & 0.069 & 0.045 & 0.054 & 0.086 & 0.033 & 0.033 \\
\hline
\end{tabular}


Table S2: Annual average particle-phase PCB concentrations at NJADN sites

\begin{tabular}{|c|c|c|c|c|c|c|c|c|c|}
\hline \multicolumn{10}{|c|}{ Annual average concentrations $\left(\mathrm{pg} / \mathrm{m}^{3}\right)$} \\
\hline $\begin{array}{l}\text { site } \\
\text { PCB congener }\end{array}$ & $\begin{array}{c}\mathrm{CC} \\
\text { particle }\end{array}$ & $\begin{array}{c}\text { DB } \\
\text { particle }\end{array}$ & $\begin{array}{c}\mathrm{JC} \\
\text { particle }\end{array}$ & $\begin{array}{c}\text { NB } \\
\text { particle }\end{array}$ & $\begin{array}{c}\text { PL } \\
\text { particle }\end{array}$ & $\begin{array}{c}\text { SH } \\
\text { particle }\end{array}$ & $\begin{array}{c}\text { TK } \\
\text { particle }\end{array}$ & $\begin{array}{c}\text { WC } \\
\text { particle }\end{array}$ & $\begin{array}{c}\mathrm{CH} \\
\text { particle }\end{array}$ \\
\hline 18 & 1.4 & 0.11 & 0.96 & 0.60 & 0.090 & 0.41 & 0.094 & 0.16 & 0.014 \\
\hline $17+15$ & 0.047 & 0 & 0.53 & 0.14 & 0 & 0.054 & 0 & 0 & 0 \\
\hline $16+32$ & 2.1 & 0.58 & 1.3 & 0.73 & 0.50 & 0.76 & 0.36 & 0.62 & 0.28 \\
\hline 31 & 2.7 & 0.51 & 1.4 & 0.66 & 0.34 & 0.49 & 0.26 & 0.48 & 0.21 \\
\hline 28 & 1.5 & 0.098 & 1.0 & 0.31 & 0.037 & 0.17 & 0.018 & 0.042 & 0 \\
\hline $21+33+53$ & 0 & 0 & 0.71 & 0.47 & 0 & 0.080 & 0 & 0 & 0 \\
\hline 22 & 1.7 & 0.41 & 0.82 & 0.74 & 0.35 & 0.56 & 0.47 & 0.48 & 0.42 \\
\hline 45 & 0.12 & 0 & 0.16 & 0.10 & 0 & 0.046 & 0 & 0 & 0 \\
\hline $52+43$ & 2.7 & 0.49 & 1.6 & 0.76 & 0.29 & 0.56 & 0.33 & 0.38 & 0.33 \\
\hline 49 & 1.1 & 0.11 & 0.73 & 0.29 & 0.092 & 0.20 & 0.093 & 0.10 & 0.078 \\
\hline $47+48$ & 0.64 & 0.13 & 0.34 & 0.16 & 0.064 & 0.052 & 0.065 & 0.074 & 0.021 \\
\hline 44 & 1.9 & 0.22 & 1.0 & 0.92 & 0.12 & 0.15 & 0.095 & 0.15 & 0.027 \\
\hline $37+42$ & 0.98 & 0.050 & 0.90 & 0.23 & 0.021 & 0.17 & 0.0072 & 0.027 & 0 \\
\hline $41+71$ & 1.4 & 0.19 & 0.77 & 0.30 & 0.15 & 0.26 & 0.19 & 0.22 & 0.23 \\
\hline 64 & 0.63 & 0.075 & 0.35 & 0.21 & 0.24 & 0.094 & 0.039 & 0.045 & 0.034 \\
\hline 74 & 0.92 & 0.041 & 0.58 & 0.30 & 0.029 & 0.091 & 0.011 & 0.031 & 0.0058 \\
\hline $70+76$ & 2.5 & 0.16 & 1.0 & 0.39 & 0.083 & 0.15 & 0.055 & 0.13 & 0.056 \\
\hline $66+95$ & 6.3 & 0.66 & 2.8 & 1.6 & 0.24 & 0.90 & 0.31 & 0.40 & 0.23 \\
\hline 91 & 0.41 & 0.0056 & 0.31 & 0.20 & 0 & 0.16 & 0.0041 & 0.0053 & 0 \\
\hline $56+60+89$ & 2.6 & 0.20 & 1.1 & 0.40 & 0.089 & 0.24 & 0.075 & 0.15 & 0.085 \\
\hline $92+84$ & 3.8 & 0.46 & 1.3 & 0.71 & 0.15 & 0.44 & 0.18 & 0.28 & 0.18 \\
\hline 101 & 4.4 & 0.49 & 1.5 & 0.75 & 0.13 & 0.45 & 0.14 & 0.24 & 0.14 \\
\hline 97 & 1.4 & 0.13 & 0.39 & 0.19 & 0.030 & 0.10 & 0.043 & 0.056 & 0.042 \\
\hline $87+81$ & 0 & 0 & 0.66 & 0.37 & 0 & 0.22 & 0 & 0 & 0 \\
\hline $85+136$ & 0.84 & 0.24 & 0.64 & 0.27 & 0.15 & 0.17 & 0.19 & 0.19 & 0.18 \\
\hline $110+77$ & 7.4 & 0.62 & 2.0 & 0.94 & 0.14 & 0.53 & 0.15 & 0.28 & 0.18 \\
\hline 82 & 1.1 & 0.078 & 0.29 & 0.11 & 0.021 & 0.060 & 0.029 & 0.041 & 0.031 \\
\hline 151 & 0.85 & 0.22 & 0.51 & 0.21 & 0.058 & 0.11 & 0.077 & 0.083 & 0.060 \\
\hline $135+144+147+124$ & 1.6 & 0.23 & 0.60 & 0.17 & 0.053 & 0.10 & 0.056 & 0.094 & 0.11 \\
\hline $149+123+107$ & 5.3 & 0.64 & 2.1 & 0.68 & 0.10 & 0.40 & 0.13 & 0.23 & 0.12 \\
\hline 118 & 0 & 0 & 1.2 & 0.42 & 0 & 0.22 & 0 & 0 & 0 \\
\hline $153+132$ & 7.5 & 0.90 & 2.9 & 0.90 & 0.17 & 0.51 & 0.16 & 0.38 & 0.18 \\
\hline $163+138$ & 12 & 1.2 & 4.1 & 1.3 & 0.22 & 0.80 & 0.26 & 0.47 & 0.29 \\
\hline $187+182$ & 1.7 & 0.36 & 0.97 & 0.29 & 0.043 & 0.19 & 0.13 & 0.090 & 0.056 \\
\hline 180 & 3.2 & 0.80 & 2.6 & 0.59 & 0.13 & 0.37 & 0.26 & 0.23 & 0.13 \\
\hline 201 & 6.3 & 0.29 & 1.3 & 0.32 & 0.080 & 0.20 & 0.15 & 0.15 & 0.073 \\
\hline $203+196$ & 5.3 & 0.50 & 1.5 & 0.36 & 0.13 & 0.25 & 0.32 & 0.22 & 0.15 \\
\hline 206 & 5.4 & 0.088 & 0.58 & 0.13 & 0.069 & 0.090 & 0.084 & 0.11 & 0.035 \\
\hline Homologue Grou & & & & & & & & & \\
\hline 3 & 10 & 1.8 & 7.6 & 3.9 & 1.3 & 2.7 & 1.2 & 1.8 & 0.92 \\
\hline 4 & 21 & 2.3 & 11 & 5.6 & 1.4 & 2.9 & 1.3 & 1.7 & 1.1 \\
\hline 5 & 25 & 2.3 & 9.0 & 4.2 & 0.71 & 2.5 & 0.83 & 1.2 & 0.82 \\
\hline 6 & 32 & 3.8 & 12 & 3.6 & 0.76 & 2.2 & 0.86 & 1.5 & 0.90 \\
\hline 7 & 12 & 2.6 & 7.4 & 1.9 & 0.37 & 1.2 & 0.67 & 0.69 & 0.39 \\
\hline 8 & 17 & 1.3 & 4.4 & 1.0 & 0.34 & 0.68 & 0.67 & 0.60 & 0.35 \\
\hline 9 & 5.4 & 0.088 & 0.58 & 0.13 & 0.069 & 0.090 & 0.084 & 0.11 & 0.035 \\
\hline
\end{tabular}


Table S3: Calculated annual average PCB dry deposition fluxes at NJADN sites DEPOSITION FLUX (ug m $\left.\mathrm{m}^{-2} \mathrm{y}^{-1}\right)$

\begin{tabular}{|c|c|c|c|c|c|c|c|c|c|}
\hline $\begin{array}{l}\text { site } \\
\text { deposition mode }\end{array}$ & $\begin{array}{c}\text { CC } \\
\text { DRY }\end{array}$ & $\begin{array}{c}\text { DB } \\
\text { DRY }\end{array}$ & $\begin{array}{c}\text { JC } \\
\text { DRY }\end{array}$ & $\begin{array}{l}\text { NB } \\
\text { DRY }\end{array}$ & $\begin{array}{c}\text { PL } \\
\text { DRY }\end{array}$ & $\begin{array}{c}\text { SH } \\
\text { DRY }\end{array}$ & $\begin{array}{c}\text { TK } \\
\text { DRY }\end{array}$ & $\begin{array}{l}\text { WC } \\
\text { DRY }\end{array}$ & $\begin{array}{c}\text { CH } \\
\text { DRY }\end{array}$ \\
\hline 18 & 0.22 & 0.018 & 0.15 & 0.094 & 0.014 & 0.064 & 0.015 & 0.025 & 0.0021 \\
\hline $17+15$ & 0.0074 & 0 & 0.084 & 0.021 & 0 & 0.0086 & 0 & 0 & 0 \\
\hline $16+32$ & 0.34 & 0.092 & 0.22 & 0.11 & 0.077 & 0.12 & 0.056 & 0.098 & 0.046 \\
\hline 31 & 0.43 & 0.081 & 0.24 & 0.10 & 0.054 & 0.077 & 0.041 & 0.076 & 0.031 \\
\hline 28 & 0.24 & 0.015 & 0.17 & 0.049 & 0.0054 & 0.027 & 0.0029 & 0.0067 & 0 \\
\hline $21+33+53$ & 0 & 0 & 0.11 & 0.074 & 0 & 0.013 & 0 & 0 & 0 \\
\hline 22 & 0.27 & 0.065 & 0.13 & 0.12 & 0.058 & 0.089 & 0.073 & 0.075 & 0.068 \\
\hline 45 & 0.019 & 0 & 0.026 & 0.017 & 0 & 0.0072 & 0 & 0 & 0 \\
\hline $52+43$ & 0.42 & 0.078 & 0.25 & 0.12 & 0.047 & 0.088 & 0.052 & 0.060 & 0.056 \\
\hline 49 & 0.18 & 0.018 & 0.12 & 0.046 & 0.015 & 0.031 & 0.015 & 0.016 & 0.013 \\
\hline $47+48$ & 0.10 & 0.021 & 0.059 & 0.026 & 0.010 & 0.0082 & 0.010 & 0.012 & 0.0032 \\
\hline 44 & 0.30 & 0.035 & 0.18 & 0.15 & 0.017 & 0.024 & 0.015 & 0.023 & 0.0037 \\
\hline $37+42$ & 0.15 & 0.0079 & 0.15 & 0.036 & 0.0031 & 0.027 & 0.0011 & 0.0043 & 0 \\
\hline $41+71$ & 0.21 & 0.030 & 0.13 & 0.048 & 0.026 & 0.042 & 0.029 & 0.035 & 0.039 \\
\hline 64 & 0.10 & 0.012 & 0.060 & 0.033 & 0.037 & 0.015 & 0.0061 & 0.0070 & 0.0055 \\
\hline 74 & 0.14 & 0.0065 & 0.095 & 0.047 & 0.0044 & 0.014 & 0.0018 & 0.0049 & 0.00064 \\
\hline $70+76$ & 0.40 & 0.026 & 0.17 & 0.062 & 0.013 & 0.023 & 0.0086 & 0.021 & 0.0091 \\
\hline $66+95$ & 1.0 & 0.10 & 0.47 & 0.25 & 0.039 & 0.14 & 0.049 & 0.063 & 0.038 \\
\hline 91 & 0.064 & 0.00088 & 0.051 & 0.031 & 0 & 0.025 & 0.00064 & 0.00083 & 0 \\
\hline $56+60+89$ & 0.40 & 0.031 & 0.18 & 0.064 & 0.015 & 0.037 & 0.012 & 0.024 & 0.014 \\
\hline $92+84$ & 0.60 & 0.073 & 0.22 & 0.11 & 0.024 & 0.069 & 0.029 & 0.044 & 0.030 \\
\hline 101 & 0.69 & 0.078 & 0.25 & 0.12 & 0.021 & 0.071 & 0.021 & 0.038 & 0.022 \\
\hline 97 & 0.22 & 0.020 & 0.065 & 0.030 & 0.0049 & 0.016 & 0.0068 & 0.0089 & 0.0070 \\
\hline $87+81$ & 0 & 0 & 0.11 & 0.058 & 0 & 0.035 & 0 & 0 & 0 \\
\hline $85+136$ & 0.13 & 0.037 & 0.11 & 0.043 & 0.024 & 0.026 & 0.031 & 0.029 & 0.030 \\
\hline $110+77$ & 1.2 & 0.098 & 0.33 & 0.15 & 0.024 & 0.083 & 0.023 & 0.044 & 0.029 \\
\hline 82 & 0.17 & 0.012 & 0.048 & 0.017 & 0.0035 & 0.0094 & 0.0045 & 0.0065 & 0.0053 \\
\hline 151 & 0.13 & 0.034 & 0.087 & 0.033 & 0.0092 & 0.017 & 0.012 & 0.013 & 0.0092 \\
\hline $135+144+147+124$ & 0.25 & 0.036 & 0.10 & 0.027 & 0.0086 & 0.016 & 0.0088 & 0.015 & 0.018 \\
\hline $149+123+107$ & 0.83 & 0.10 & 0.35 & 0.11 & 0.017 & 0.064 & 0.020 & 0.036 & 0.020 \\
\hline 118 & 0 & 0 & 0.19 & 0.067 & 0 & 0.035 & 0 & 0 & 0 \\
\hline $153+132$ & 1.2 & 0.14 & 0.49 & 0.14 & 0.028 & 0.080 & 0.024 & 0.059 & 0.030 \\
\hline $163+138$ & 2.0 & 0.19 & 0.69 & 0.20 & 0.037 & 0.13 & 0.041 & 0.074 & 0.047 \\
\hline $187+182$ & 0.27 & 0.057 & 0.17 & 0.046 & 0.0069 & 0.029 & 0.021 & 0.014 & 0.0052 \\
\hline 180 & 0.50 & 0.13 & 0.44 & 0.093 & 0.022 & 0.059 & 0.041 & 0.036 & 0.021 \\
\hline 201 & 1.0 & 0.045 & 0.21 & 0.051 & 0.013 & 0.032 & 0.024 & 0.024 & 0.012 \\
\hline $203+196$ & 0.84 & 0.079 & 0.25 & 0.057 & 0.020 & 0.039 & 0.050 & 0.034 & 0.016 \\
\hline 206 & 0.85 & 0.014 & 0.092 & 0.021 & 0.011 & 0.014 & 0.013 & 0.018 & 0.0058 \\
\hline \multicolumn{10}{|l|}{ Homologue Group } \\
\hline 3 & 1.7 & 0.28 & 1.3 & 0.61 & 0.21 & 0.43 & 0.19 & 0.29 & 0.15 \\
\hline 4 & 3.3 & 0.36 & 1.8 & 0.89 & 0.22 & 0.45 & 0.20 & 0.27 & 0.18 \\
\hline 5 & 3.7 & 0.36 & 1.5 & 0.66 & 0.11 & 0.40 & 0.13 & 0.19 & 0.13 \\
\hline 6 & 4.8 & 0.61 & 2.0 & 0.57 & 0.13 & 0.35 & 0.14 & 0.24 & 0.15 \\
\hline 7 & 1.7 & 0.40 & 1.3 & 0.29 & 0.059 & 0.19 & 0.10 & 0.10 & 0.056 \\
\hline 8 & 2.7 & 0.20 & 0.74 & 0.16 & 0.056 & 0.11 & 0.11 & 0.094 & 0.051 \\
\hline 9 & 0.85 & 0.014 & 0.092 & 0.021 & 0.011 & 0.014 & 0.013 & 0.018 & 0.0058 \\
\hline
\end{tabular}


Table S4: Calculated annual average PCB gas absorption fluxes at NJADN sites DEPOSITION FLUX (ug $\left.\mathbf{m}^{-2} \mathrm{y}^{-1}\right)$

\begin{tabular}{|c|c|c|c|c|c|c|c|c|c|}
\hline $\begin{array}{l}\text { site } \\
\text { deposition mode }\end{array}$ & $\begin{array}{l}\text { CC } \\
\text { GAS }\end{array}$ & $\begin{array}{l}\text { DB } \\
\text { GAS }\end{array}$ & $\begin{array}{c}\text { JC } \\
\text { GAS }\end{array}$ & $\begin{array}{l}\text { NB } \\
\text { GAS }\end{array}$ & $\begin{array}{l}\text { PL } \\
\text { GAS }\end{array}$ & $\begin{array}{l}\text { SH } \\
\text { GAS }\end{array}$ & $\begin{array}{l}\text { TK } \\
\text { GAS }\end{array}$ & $\begin{array}{l}\text { WC } \\
\text { GAS }\end{array}$ & $\begin{array}{l}\text { CH } \\
\text { GAS }\end{array}$ \\
\hline 18 & 6.4 & 0.045 & 4.0 & 0.33 & 0.19 & 1.5 & 0.38 & 0.58 & 0.11 \\
\hline $17+15$ & 3.2 & 0.00093 & 3.1 & 0.17 & 0.13 & 0.72 & 0.19 & 0.25 & 0.034 \\
\hline $16+32$ & 7.1 & 0.044 & 4.7 & 0.47 & 0.21 & 1.8 & 0.58 & 0.66 & 0.12 \\
\hline 31 & 8.1 & 0.039 & 3.4 & 0.30 & 0.15 & 1.2 & 0.30 & 0.47 & 0.078 \\
\hline 28 & 4.1 & 0.016 & 2.2 & 0.17 & 0.078 & 0.81 & 0.17 & 0.23 & 0.040 \\
\hline $21+33+53$ & 3.0 & 0.0061 & 2.0 & 0.19 & 0.056 & 0.54 & 0.15 & 0.17 & 0.024 \\
\hline 22 & 3.3 & 0.013 & 0.98 & 0.15 & 0.067 & 0.59 & 0.16 & 0.22 & 0.046 \\
\hline 45 & 0.36 & 0.0012 & 0.37 & 0.033 & 0.010 & 0.16 & 0.015 & 0.028 & 0.0053 \\
\hline $52+43$ & 7.7 & 0.023 & 2.3 & 0.22 & 0.11 & 1.3 & 0.28 & 0.34 & 0.064 \\
\hline 49 & 1.9 & 0.014 & 0.97 & 0.094 & 0.072 & 0.56 & 0.15 & 0.22 & 0.044 \\
\hline $47+48$ & 1.3 & 0.0054 & 0.67 & 0.10 & 0.032 & 0.53 & 0.073 & 0.090 & 0.016 \\
\hline 44 & 4.7 & 0.015 & 1.7 & 0.15 & 0.076 & 0.77 & 0.16 & 0.33 & 0.039 \\
\hline $37+42$ & 2.8 & 0.012 & 1.7 & 0.13 & 0.073 & 0.70 & 0.15 & 0.21 & 0.050 \\
\hline $41+71$ & 1.6 & 0.0043 & 0.78 & 0.054 & 0.026 & 0.35 & 0.061 & 0.077 & 0.018 \\
\hline 64 & 0.88 & 0.0022 & 0.37 & 0.031 & 0.015 & 0.16 & 0.031 & 0.046 & 0.0076 \\
\hline 74 & 1.1 & 0.0037 & 0.31 & 0.038 & 0.013 & 0.18 & 0.031 & 0.041 & 0.0077 \\
\hline $70+76$ & 3.2 & 0.0073 & 0.72 & 0.080 & 0.032 & 0.38 & 0.071 & 0.096 & 0.019 \\
\hline $66+95$ & 8.6 & 0.028 & 2.1 & 0.24 & 0.094 & 1.2 & 0.23 & 0.27 & 0.055 \\
\hline 91 & 0.46 & 0.00084 & 0.15 & 0.017 & 0.0048 & 0.11 & 0.0072 & 0.014 & 0.0020 \\
\hline $56+60+89$ & 2.5 & 0.0082 & 0.68 & 0.068 & 0.028 & 0.33 & 0.073 & 0.085 & 0.017 \\
\hline $92+84$ & 2.6 & 0.011 & 0.71 & 0.065 & 0.036 & 0.33 & 0.087 & 0.099 & 0.018 \\
\hline 101 & 3.6 & 0.013 & 0.73 & 0.079 & 0.040 & 0.39 & 0.094 & 0.10 & 0.023 \\
\hline 97 & 0.74 & 0.0027 & 0.15 & 0.017 & 0.0085 & 0.088 & 0.019 & 0.021 & 0.0050 \\
\hline $87+81$ & 1.3 & 0.0069 & 0.34 & 0.044 & 0.021 & 0.16 & 0.059 & 0.043 & 0.0080 \\
\hline $85+136$ & 0.51 & 0.0023 & 0.16 & 0.046 & 0.0046 & 0.079 & 0.012 & 0.014 & 0.0027 \\
\hline $110+77$ & 4.0 & 0.016 & 0.74 & 0.096 & 0.033 & 0.42 & 0.083 & 0.098 & 0.024 \\
\hline 82 & 0.36 & 0.0012 & 0.057 & 0.0069 & 0.0028 & 0.032 & 0.0058 & 0.0081 & 0.0020 \\
\hline 151 & 0.23 & 0.0037 & 0.085 & 0.0077 & 0.0055 & 0.039 & 0.015 & 0.014 & 0.0033 \\
\hline $135+144+147+124$ & 0.39 & 0.0038 & 0.10 & 0.010 & 0.0060 & 0.050 & 0.014 & 0.014 & 0.0057 \\
\hline $149+123+107$ & 1.1 & 0.012 & 0.29 & 0.027 & 0.012 & 0.13 & 0.034 & 0.036 & 0.0090 \\
\hline 118 & 1.3 & 0.011 & 0.27 & 0.040 & 0.0092 & 0.12 & 0.043 & 0.028 & 0.0056 \\
\hline $153+132$ & 1.5 & 0.032 & 0.42 & 0.045 & 0.019 & 0.19 & 0.056 & 0.054 & 0.015 \\
\hline $163+138$ & 2.1 & 0.058 & 0.50 & 0.062 & 0.022 & 0.22 & 0.067 & 0.062 & 0.022 \\
\hline $187+182$ & 0.30 & 0.020 & 0.11 & 0.018 & 0.0056 & 0.064 & 0.023 & 0.016 & 0.0038 \\
\hline 180 & 0.32 & 0.082 & 0.17 & 0.025 & 0.0094 & 0.058 & 0.025 & 0.024 & 0.0075 \\
\hline 201 & 0.31 & 0.019 & 0.042 & 0.0078 & 0.0027 & 0.017 & 0.013 & 0.0090 & 0.0019 \\
\hline $203+196$ & 0.84 & 0.044 & 0.11 & 0.020 & 0.0099 & 0.045 & 0.034 & 0.024 & 0.0078 \\
\hline 206 & 0.12 & 0.0034 & 0.0104 & 0.0016 & 0.0011 & 0.0044 & 0.0042 & 0.0023 & 0.00069 \\
\hline \multicolumn{10}{|l|}{ Homologue Group } \\
\hline 3 & 38 & 0.10 & 22 & 1.9 & 0.95 & 7.9 & 2.1 & 2.8 & 0.49 \\
\hline 4 & 34 & 0.098 & 11 & 1.1 & 0.51 & 6.0 & 1.2 & 1.6 & 0.29 \\
\hline 5 & 16 & 0.032 & 3.5 & 0.43 & 0.17 & 1.8 & 0.43 & 0.45 & 0.096 \\
\hline 6 & 6.0 & 0.031 & 1.6 & 0.18 & 0.083 & 0.72 & 0.23 & 0.23 & 0.067 \\
\hline 7 & 1.2 & 0.022 & 0.66 & 0.089 & 0.031 & 0.25 & 0.11 & 0.098 & 0.025 \\
\hline 8 & 1.7 & 0.022 & 0.28 & 0.044 & 0.021 & 0.098 & 0.078 & 0.057 & 0.016 \\
\hline 9 & 0.12 & 0.0011 & 0.011 & 0.0016 & 0.0011 & 0.0044 & 0.0042 & 0.0023 & 0.00069 \\
\hline
\end{tabular}

